\title{
Energy loss of charged particles interacting with simple metal surfaces
}

\author{
A. García-Lekue ${ }^{1}$ and J. M. Pitarke ${ }^{1,2}$ \\ ${ }^{1}$ Materia Kondentsatuaren Fisika Saila, Zientzi Fakultatea, Euskal Herriko Unibertsitatea, \\ 644 Posta kutxatila, 48080 Bilbo, Basque Country, Spain \\ ${ }^{2}$ Donostia International Physics Center (DIPC) and Centro Mixto CSIC-UPV/EHU, \\ Basque Country, Spain
}

(4 January 2001)

\begin{abstract}
Self-consistent calculations of the energy-loss spectra of charged particles moving near a planebounded free electron gas are reported. Energy-loss probabilities are obtained, within linear-response theory, from the knowledge of the density-response function of the inhomogeneous electron system. Self-consistent single-particle wave functions and energies are obtained by solving the Kohn-Sham equation of density-functional theory, and the electronic response is then computed either in the random-phase approximation or with the use of an adiabatic local-density approximation. Special emphasis is placed on the various contributions from collective and electron-hole excitations to the energy loss of charged particles moving parallel with the surface. The effect of the electronic selvage at a metal surface on the energy-loss spectra is also discussed, by comparing our full self-consistent calculations with those obtained for electron densities that drop abruptly to zero at the surface.
\end{abstract}

\section{INTRODUCTION}

Charged particles interacting with metal surfaces create electron-hole pairs and, by virtue of the dynamically screened Coulomb interaction bulk and surface collective excitations, i.e., plasmons. play a crucial role in the interpretation of surface electron spectroscopy data, such as x-ray photoelectron spectroscopy (XPS), Auger-electron spectroscopy (AES), and reflection-electron energy-loss spectroscopy (REELS). The interaction of swift electrons with surfaces has also attracted great interest in the field of scanning transmission electron microscopy (STEM) 1 G Equally, the interaction of moving ions with solids has represented an active field of basic and applied physics, 19 and a great amount of research has recently been focused on the case of ions that are incident at grazing angle.1913 Nevertheless, existing calculations of energy-loss spectra invoke either the local-dielectric, the hydrodynamic, or the specular-reflexion model of the surface.14 21 An exception is a recent self-consistent calculation of the stopping power of jellium planar surfaces for ions moving parallel with the surface.22

In this paper, we present extensive self-consistent calculations of the energy-loss spectra of charged particles moving near a jellium surface. In the case of charged particles moving inside a solid, nonlinear effects are known to be crucial in the interpretation of energy-loss measurements;23.24 however, these corrections have been shown to be less impgrtant when the charged particle moves outside the solid 25 In Section II we present, within first-order perturbation [or, equivalently, linear-response] theory, general expressions for the energy-loss probability of charged particles moving along a definite trajectory in inhomogeneous media, and focus on the case of a bounded three-dimensional electron gas that is translationally invariant in the plane of the surface. In Section
III, we report the results of our full self-consistent calculations of energy-loss spectra of charged particles moving parallel with the surface, which are found to satisfy sumrules for particle-number conservation. Special emphasis is placed on the various contributions from collective and electron-hole excitations to the energy-loss probability. The effect of the electronic selvage at a metal surface on the energy-loss spectra is also discussed, by comparing our full self-consistent calculations with those obtained for electron densities that drop abruptly to zero at the surface. In Section IV our conclusions are presented.

Unless otherwise is stated, we use atomic units throughout, i.e., $e^{2}=\hbar=m_{e}=1$.

\section{THEORY}

We consider a recoiless particle of charge $Z_{1}$ moving in an arbitrary inhomogeneous electron system at a given impact vector $\mathbf{b}$ with non-relativistic velocity $\mathbf{v}$, for which retardation effects and radiation losses can be neglected 26 Within first-order perturbation theory, the probability for the probe particle to transfer momentum $\mathbf{q}$ to the medium is given by the following expression:27

$$
\begin{aligned}
P_{\mathbf{q}}= & -\frac{4 \pi}{L A} Z_{1}^{2} \int_{0}^{\infty} d \omega \int \frac{d \mathbf{q}^{\prime}}{(2 \pi)^{3}} \mathrm{e}^{i \mathbf{b} \cdot\left(\mathbf{q}+\mathbf{q}^{\prime}\right)} \\
& \times \operatorname{Im} W\left(\mathbf{q}, \mathbf{q}^{\prime} ; \omega\right) \delta(\omega-\mathbf{q} \cdot \mathbf{v}) \delta\left(\omega+\mathbf{q}^{\prime} \cdot \mathbf{v}\right),
\end{aligned}
$$

where $L$ and $A$ represent the normalization length and area, respectively, and $W\left(\mathbf{q}, \mathbf{q}^{\prime} ; \omega\right)$ is the screened interaction

$$
W\left(\mathbf{q}, \mathbf{q}^{\prime} ; \omega\right)=\int d \mathbf{r} \int d \mathbf{r}^{\prime} \mathrm{e}^{-i\left(\mathbf{q} \cdot \mathbf{r}+\mathbf{q}^{\prime} \cdot \mathbf{r}^{\prime}\right)} W\left(\mathbf{r}, \mathbf{r}^{\prime} ; \omega\right),
$$

with 


$$
\begin{array}{r}
W\left(\mathbf{r}, \mathbf{r}^{\prime} ; \omega\right)=v\left(\mathbf{r}, \mathbf{r}^{\prime}\right)+\int d \mathbf{r}_{1} \int d \mathbf{r}_{2} \\
\times v\left(\mathbf{r}, \mathbf{r}_{1}\right) \chi\left(\mathbf{r}_{1}, \mathbf{r}_{2}, \omega\right) v\left(\mathbf{r}_{2}, \mathbf{r}^{\prime}\right) .
\end{array}
$$

Here, $v\left(\mathbf{r}, \mathbf{r}^{\prime}\right)$ represents the bare Coulomb interaction and $\chi\left(\mathbf{r}, \mathbf{r}^{\prime}, \omega\right)$ is the so-called density-response function of the medium. 28

Within a self-energy formalism, the decay rate of the probe particle is obtained from the knowledge of the imaginary part of the self-energy. In the GW approximation, and replacing the probe-particle Green function by that of a non-interacting recoiless particle, one finds:29

$$
\begin{aligned}
\tau^{-1}= & -2 Z_{1}^{2} \sum_{f} \int d \mathbf{r} \int d \mathbf{r}^{\prime} \phi_{i}^{*}(\mathbf{r}) \phi_{f}^{*}\left(\mathbf{r}^{\prime}\right) \\
& \times \operatorname{Im} W\left(\mathbf{r}, \mathbf{r}^{\prime}, E_{i}-E_{f}\right) \phi_{i}\left(\mathbf{r}^{\prime}\right) \phi_{f}(\mathbf{r}),
\end{aligned}
$$

where $\phi_{i}(\mathbf{r})$ represents the probe-particle initial state of energy $E_{i}$, and the sum is extended over a complete set of final states $\phi_{f}(\mathbf{r})$ of energy $E_{f}$. Describing the probeparticle initial and final states by plane waves in the direction of motion and a Dirac $\delta$ function in the transverse direction, i.e.,

$$
\phi(\mathbf{r})=\frac{1}{\sqrt{A}} e^{i \mathbf{v} \cdot \mathbf{r}} \sqrt{\delta\left(\mathbf{r}_{\perp}-\mathbf{b}\right)},
$$

where $\mathbf{r}_{\perp}$ represents the position vector perpendicular to the projectile velocity, one finds

$$
\tau^{-1}=\frac{1}{T} \sum_{\mathbf{q}} P_{\mathbf{q}}
$$

$T$ being the normalization time and $P_{\mathbf{q}}$ the probability for the probe particle to transfer momentum $\mathbf{q}$ to the medium, as obtained from Eq. (1).

Alternatively, one may consider the energy that the probe particle looses per unit time due to electronic excitations in the medium. This can be written as 30

$$
-\frac{d E}{d t}=-\int d \mathbf{r} \rho^{e x t}(\mathbf{r}, t) \frac{\partial V^{i n d}(\mathbf{r}, t)}{\partial t},
$$

where $\rho^{e x t}(\mathbf{r}, t)$ represents the probe-particle charge density

$$
\rho^{e x t}(\mathbf{r}, t)=Z_{1} \delta(\mathbf{r}-\mathbf{b}-\mathbf{v} t)
$$

and $V^{i n d}(\mathbf{r}, t)$ is the induced potential. To first order in $\rho^{e x t}(\mathbf{r}, t)$, i.e., within linear-response theory, one finds

$$
\begin{aligned}
V^{i n d}(\mathbf{r}, t)=\int & d \mathbf{r}^{\prime} \int_{-\infty}^{+\infty} d t^{\prime} \int_{-\infty}^{+\infty} \frac{d \omega}{2 \pi} \mathrm{e}^{-i \omega\left(t-t^{\prime}\right)} \\
& \times\left[W\left(\mathbf{r}, \mathbf{r}^{\prime}, \omega\right)-v\left(\mathbf{r}, \mathbf{r}^{\prime}\right)\right] \rho^{e x t}\left(\mathbf{r}^{\prime}, t^{\prime}\right) .
\end{aligned}
$$

Introduction of Eqs. (8) and (9) into Eq. (7) yields the total energy lost by the particle

$$
-\Delta E=\int_{-\infty}^{+\infty} d t\left(-\frac{d E}{d t}\right)=\sum_{\mathbf{q}}(\mathbf{q} \cdot \mathbf{v}) P_{\mathbf{q}}
$$

where $P_{\mathbf{q}}$ is, as in Eq. (6), the probability of Eq. (1) for the probe particle to transfer momentum $\mathbf{q}$ to the medium, and $\mathbf{q} \cdot \mathbf{v}$ represents the corresponding energy transfer.

The results in Eqs. (何), (6), and (10) are general expressions for the case of a classical trajectory in an arbitrary inhomogeneous electron system characterized by the density-response function $\chi\left(\mathbf{r}, \mathbf{r}^{\prime} ; \omega\right)$. In particular, in the case of a bounded three-dimensional electron gas that is translationally invariant in two directions, which we take to be normal to the $z$ axis, the energy loss of Eq. (10) may be expressed in terms of the two-dimensional Fourier transform of the screened interaction, as follows

$$
\begin{aligned}
-\Delta E= & -\frac{Z_{1}^{2}}{\pi} \int \frac{d \mathbf{q}_{\|}}{(2 \pi)^{2}} \int_{-\infty}^{+\infty} d t \int_{-\infty}^{+\infty} d t^{\prime} \int_{0}^{\infty} d \omega \omega \\
& \times \mathrm{e}^{-i\left(\omega-\mathbf{q}_{\|} \cdot \mathbf{v}_{\|}\right)\left(t-t^{\prime}\right)} \operatorname{Im} W\left[z(t), z\left(t^{\prime}\right) ; \mathbf{q}_{\|}, \omega\right],
\end{aligned}
$$

where $\mathbf{q}_{\|}$and $\mathbf{v}_{\|}$are the momentum transfer and the velocity in the plane of the surface and $z(t)$ represents the position of the projectile relative to the surface. Eq. (11) gives the energy that a charged particle moving with constant velocity along an arbitrary trajectory looses due to electronic excitations in an electron system that is translationally invariant in two directions, as occurs in the case of a simple metal surface modeled by jellium.

\section{A. Parallel trajectory}

In the glancing incidence geometry ions penetrate into the material, they skim the outermost layer of the solid, and are then repelled by a repulsive, screened Coulomb potential, as discussed by Gemmell 31 Through use of the appropriate effective potentials the ion trajectory $z(t)$ can be calculated and the energy loss is then obtained from Eq. (11). Here we restrict our attention to the case of charged particles moving with constant velocity $\mathbf{v}$ along a definite trajectory at a fixed distance $z$ from a jellium surface, as approximately occurs under extreme grazing-incidence conditions. Eq. (11) then yields

$$
-\Delta E=L\left(-\frac{d E}{d x}\right)
$$

where $(-d E / d x)$ is the energy loss per unit path length of the projectile, i.e., the so-called stopping power of the electron system,

$$
\begin{aligned}
-\frac{d E}{d x}= & -\frac{2}{v} Z_{1}^{2} \int \frac{d \mathbf{q}_{\|}}{(2 \pi)^{2}} \int_{0}^{\infty} d \omega \omega \\
& \times \operatorname{Im} W\left(z, z ; \mathbf{q}_{\|}, \omega\right) \delta\left(\omega-\mathbf{q}_{\|} \cdot \mathbf{v}\right)
\end{aligned}
$$


Eq. (13) can be expressed in terms of $P\left(q_{\|}, \omega\right)$, which represents the probability per unit time, unit wave number and unit frequency for the probe particle to transfer momentum $q_{\|}$and energy $\omega$ to the medium:

$$
-\frac{d E}{d x}=\frac{1}{v} \int_{0}^{\infty} d q_{\|} \int_{0}^{q_{\|} v} d \omega \omega P\left(q_{\|}, \omega\right),
$$

where

$$
P\left(q_{\|}, \omega\right)=-\frac{Z_{1}^{2}}{\pi^{2} v} \operatorname{Im} W\left(z, z ; q_{\|}, \omega\right) \frac{q_{\|}}{\sqrt{q_{\|}^{2}-(\omega / v)^{2}}} .
$$

Alternatively, the stopping power of the system is often described by means of $P(\omega)$, the total probability of exchanging energy $\omega$ with the medium:

$$
-\frac{d E}{d x}=\frac{1}{v} \int_{0}^{\infty} d \omega \omega P(\omega),
$$

where

$$
P(\omega)=-\frac{Z_{1}^{2}}{\pi^{2} v} \int_{0}^{\infty} d q_{x} \operatorname{Im} W\left(z, z ; q_{\|}, \omega\right),
$$

with $q_{\|}=\sqrt{q_{x}^{2}+(\omega / v)^{2}}$.

The main ingredient in the evaluation of Eqs. (15) and (17) is the screened interaction $W\left(z, z^{\prime} ; q_{\|}, \omega\right)$. From Eq. (3), one easily finds

$$
\begin{aligned}
& W\left(z, z^{\prime} ; q_{\|}, \omega\right)=v\left(z, z^{\prime}, q_{\|}\right)+\int d z_{1} \int d z_{2} \\
& \quad \times v\left(z, z_{1} ; q_{\|}\right) \chi\left(z_{1}, z_{2} ; q_{\|}, \omega\right) v\left(z_{2}, z^{\prime} ; q_{\|}\right),
\end{aligned}
$$

where $v\left(z, z^{\prime} ; q_{\|}\right)$and $\chi\left(z, z^{\prime} ; q_{\|}, \omega\right)$ are two-dimensional Fourier transforms of the bare Coulomb interaction and the density-response function, respectively.

In particular, for $z$ and $z^{\prime}$ coordinates that are well inside the solid, there is translational invariance in the direction normal to the surface and $W\left(z, z^{\prime} ; q_{\|}, \omega\right)$ can then be easily obtained as follows

$$
W\left(z, z^{\prime} ; q_{\|}, \omega\right)=\int_{0}^{\infty} d q_{z} e^{i q_{z}\left(z-z^{\prime}\right)} v(q) \epsilon^{-1}(q, \omega),
$$

where $q=\sqrt{q_{\|}^{2}+q_{z}^{2}}$ and $\epsilon^{-1}(q, \omega)$ represents the inverse dielectric function of a uniform electron gas,

$$
\epsilon^{-1}(q, \omega)=1+v(q) \chi(q, \omega),
$$

$v(q)$ and $\chi(\mathbf{q}, \omega)$ being three-dimensional Fourier transforms of the bare Coulomb interaction and the densityresponse function, respectively.

For $z$ and $z^{\prime}$ coordinates that are far from the surface into the vacuum, where the electron density vanishes, Eq. (18) yields

$$
W\left(z, z^{\prime} ; q_{\|}, \omega\right)=v\left(z, z^{\prime} ; q_{\|}\right)-\frac{2 \pi}{q_{\|}} e^{-q_{\|}\left(z+z^{\prime}\right)} g\left(q_{\|}, \omega\right),
$$

where $g\left(q_{\|}, \omega\right)$ is the so-called surface-response function

$$
g\left(q_{\|}, \omega\right)=-\frac{2 \pi}{q_{\|}} \int d z_{1} \int d z_{2} e^{q_{\|}\left(z_{1}+z_{2}\right)} \chi\left(z_{1}, z_{2} ; q_{\|}, \omega\right) .
$$

The energy-loss function $\operatorname{Im} g\left(q_{\|}, \omega\right)$ satisfies the $f$ sumrule 32

$$
\int_{0}^{\infty} d \omega \omega \operatorname{Im} g\left(q_{\|}, \omega\right)=2 \pi^{2} q_{\|} \int_{-\infty}^{\infty} d z \mathrm{e}^{2 q_{\|} z} n(z),
$$

which applies to the case of a bounded three-dimensional electron gas whose exact density in the ground state is $n(z)$. For $z$ coordinates that are well inside the solid the electron density takes a constant value $\bar{n}$, and for $z$ coordinates that are far from the surface into the vacuum the electron density vanishes.

In the long-wavelength limit $\left(q_{\|} \rightarrow 0\right)$,

$$
\operatorname{Im} g\left(q_{\|}, \omega\right) \rightarrow \frac{\pi}{2} \omega_{s} \delta\left(\omega-\omega_{s}\right),
$$

where $\omega_{s}=\omega_{p} / \sqrt{2}$ and $\omega_{p}=(4 \pi \bar{n})^{1 / 2}$ is the classical plasma frequency of a uniform electron gas of density $\bar{n}$. Hence, in the $q_{\|} \rightarrow 0$ limit the energy loss is dominated by the excitation of surface plasmons of energy $\omega_{s}$, as predicted by Ritchie. 3

\section{B. The density-response function}

We consider a jellium slab of thickness $a$ normal to the $z$ axis, consisting of a fixed uniform positive background of density

$$
n_{+}(z)= \begin{cases}\bar{n}, & -a \leq z \leq 0 \\ 0, & \text { elsewhere }\end{cases}
$$

plus a neutralizing cloud of interacting electrons of density $n(z)$. The positive-background charge density $\bar{n}$ is often expressed in terms of the Wigner radius $r_{s}$, as $1 / \bar{n}=(4 \pi / 3) r_{s}^{3}$.

Time-dependent density-functional theory (TDDFT) shows that the exact density-response function of the electron system satisfies the integral equation 33

$$
\begin{aligned}
& \chi\left(z, z^{\prime} ; q_{\|}, \omega\right)=\chi^{0}\left(z, z^{\prime} ; q_{\|}, \omega\right)+\int d z_{1} \int \mathrm{d} z_{2} \\
& \quad \times \chi^{0}\left(z, z^{\prime} ; q_{\|}, \omega\right)\left[v\left(z_{1}, z_{2} ; q_{\|}\right)+f_{x c}\left(z_{1}, z_{2} ; q_{\|}, \omega\right)\right] \\
& \quad \times \chi\left(z_{2}, z^{\prime} ; q_{\|}, \omega\right),
\end{aligned}
$$

where $\chi^{0}\left(z, z^{\prime} ; q_{\|}, \omega\right)$ is the density-response function of non-interacting Kohn-Sham electrons

$$
\chi^{0}\left(z, z^{\prime} ; q_{\|}, \omega\right)=2 \sum_{i, j} \phi_{i}(z) \phi_{j}^{*}(z) \phi_{j}\left(z^{\prime}\right) \phi_{i}^{*}\left(z^{\prime}\right)
$$




$$
\times \int \frac{\mathrm{d} \mathbf{k}_{\|}}{(2 \pi)^{2}} \frac{\Theta\left(E_{F}-E_{i}\right)-\Theta\left(E_{F}-E_{j}\right)}{E_{i}-E_{j}+(\omega+i \eta)},
$$

and the kernel $f_{x c}\left(z, z^{\prime} ; q_{\|}, \omega\right)$ accounts for exchangecorrelation (xc) effects beyond a time-dependent Hartree approximation. In Eq. (27), $\Theta(x)$ is the Heaviside step function, $\eta$ is a positive infinitesimal, the energies $E_{i}$ and $E_{j}$ are

$$
E_{i}=\varepsilon_{i}+\frac{\mathbf{k}_{\|}^{2}}{2}
$$

and

$$
E_{j}=\varepsilon_{j}+\frac{\left(\mathbf{k}_{\|}+\mathbf{q}_{\|}\right)^{2}}{2},
$$

and the wave functions $\phi_{i}(z)$ and energies $\varepsilon_{i}$, which describe motion normal to the surface, are the eigenfunctions and eigenvalues of the one-dimensional Kohn-Sham Hamiltonian

$$
H=-\frac{1}{2} \frac{d^{2}}{z^{2}}+\varphi(z)+v_{x c}(z),
$$

$\varphi(z)$ being the electrostatic potential and $v_{x c}(z)$ representing the so-called xc potential of density-functional theory (DFT). 34

Within this scheme, the simplest possible approximation is to neglect $\mathrm{xc}$ altogether and set the xc potential $v_{x c}(z)$ and the xc kernel $f_{x c}\left(z, z^{\prime} ; q_{\|}, \omega\right)$ equal to zero. In this case, the one-dimensional single-particle wave functions and energies are the self-consistent eigenfunctions and eigenvalues of the one-electron Hartree Hamiltonian. The calculation of the density-response function is further simplified if the self-consistent electrostatic potential entering Eq. (30) is replaced by

$$
\varphi(z)= \begin{cases}0, & -a-z_{0} \leq z \leq z_{0} \\ \infty, & \text { elsewhere, }\end{cases}
$$

where $z_{0}$ is chosen so as to ensure charge neutrality. This is the so-called infinite-barrier model (IBM).35 Within this model, the one-electron wave functions are simply sines, and charge neutrality is easily found to yield

$$
z_{0}=(3 / 16) \lambda_{F}\left[1+O\left(\lambda_{F} / a\right)\right] .
$$

Exchange-correlation effects are usually introduced within the local-density approximation (LDA) of DFT, by replacing the xc potential at $z$ by that of a uniform electron gas with the local density $n(z)$. The xc kernel entering Eq. (26) is then set either equat to zero [this is the random-phase approximation (RPA) 3 ] or equal to the static $(\omega=0)$ xc kernel

$$
f_{x c}^{A L D A}\left(z, z^{\prime} ; q_{\|}, \omega\right)=\left[\frac{d v_{x c}(n)}{d n}\right]_{n=n(z)} \delta\left(z-z^{\prime}\right) .
$$

This is the so-called adiabatic local-density approximation (ALDA) 37

To compute the interacting density-response function $\chi\left(z, z^{\prime} ; q_{\|}, \omega\right)$, we follow the method described in Ref. 38. We first assume that $g(z)$ vanishes at a distance $z_{0}$ from either jellium edge, 39 and expand the wave functions $\phi_{i}(z)$ in a Fourier sine series. We then introduce a double-cosine Fourier representation for the densityresponse function, and find explicit expressions for the screened interaction and the surface-response function in terms of the Fourier coefficients of the density-response function (see Appendix A).

Great care was exercised to ensure that our slab calculations are a faithful representation of the screened interaction and the energy-loss probability in a semi-infinite medium. This issue is important, in view of the significant quantum-size effects (QSE) 10 originated in the quantization of the energy levels normal to the surface: as the slab-thickness $a$ increases new subbands for the $z$ motion become occupied, thereby leading to oscillatory functions of $a$ (the amplitude of these oscillations decays approximately linearly with $a$, and their period equals $\lambda_{F} / 2, \lambda_{F}=2 \pi /\left(3 \pi^{2} \bar{n}\right)^{1 / 3}$ being the Fermi wavelength). For each quantity $\alpha$ under study we considered three different values of $a$. One such value is the threshold width $a_{n}$ for which the $n$th subband for the $z$ motion is first occupied. The other two values are $a_{n}^{-}=a_{n}-\lambda_{F} / 4$ and $a_{n}^{+}=a_{n}+\lambda_{F} / 4$, and the infinite-width limit is then extrapolated with the use of the following relation 4142

$$
\alpha=\frac{\alpha\left(a_{n}^{-}\right)+\alpha\left(a_{n}\right)+\alpha\left(a_{n}^{+}\right)}{3} .
$$

Following this procedure to calculate the surfaceresponse function $g\left(q_{\|}, \omega\right)$, we have been able to prove that the sum-rule of Eq. (23) is satisfied for all values of $q_{\|}$under consideration. The results presented below correspond to slabs with $n=12$, for which $a \approx 5-6 \lambda_{F}$.

\section{Simplified models}

For comparison, we also consider various simplified models for the screened interaction $W\left(z, z^{\prime} ; q_{\|}, \omega\right)$ of a semi-infinite free-electron gas, which are all derived for electron densities that drop abruptly to zero at the surface. These are: (a) a classical model consisting of a semiinfinite medium of local dielectric function $\epsilon(\omega)$, (b) semiclassical and quantized hydrodynamic (HD) models, 43,44 and (c) a classical infinite barrier (CIB) or specularreflexion (SR) model 15,46 which has the virtue of incorporating dispersion effects by expressing the screened interaction in terms of the bulk dielectric function $\epsilon(q, \omega)$. Within these models and for $z$ and $z^{\prime}$ coordinates that are outside the surface $\left(z, z^{\prime}>0\right)$ the screened interaction is obtained through Eq. (21), from the knowledge of approximate expressions for the surface-response function $g\left(q_{\|}, \omega\right)$. 


\section{Classical model}

Within this approach, the screened interaction is derived by imposing the ordinary boundary conditions of continuity of the potential and the normal component of the displacement vector at the surface $(z=0)$. For $z, z^{\prime}>0$, one then easily finds Eq. (21) with

$$
g(\omega)=\frac{\epsilon(\omega)-1}{\epsilon(\omega)+1} .
$$

For a free-electron gas, the long-wavelength $(q \rightarrow 0)$ dielectric function is

$$
\epsilon(\omega)=1-\frac{\omega_{p}^{2}}{\omega(\omega+i \eta)}
$$

and introduction of Eq. (36) into Eq. (35) yields the long-wavelength limit of Eq. (24). Introducing this limit into either Eq. (15) or Eq. (17), one easily reproduces the classical expression of Echenique and Pendry 14 for the stopping power of a jellium surface,

$$
-\frac{d E}{d x}=Z_{1}^{2} \frac{\omega_{s}^{2}}{v^{2}} \mathrm{~K}_{0}\left(2 \omega_{s} z / v\right),
$$

where $K_{0}$ is the zero-order modified Bessel function. For large values of $z\left(z>>v / \omega_{s}\right)$, Eq. 37 reduces to

$$
-\frac{d E}{d x}=Z_{1}^{2} \frac{\omega_{s}}{2 v} \sqrt{\pi \omega_{s} / z v} e^{-2 \omega_{s} z / v} .
$$

\section{Hydrodynamic models}

In a HD model, the collective motion of electrons in an arbitrary inhomogeneous system is expressed in terms of the deviations from the equilibrium density. In a semiclassical approach, one writes and linearizes the basic hydrodynamic equations, i.e., the continuity and the Bernuilli equation, and for a semi-infinite system finds

$$
g\left(q_{\|}, \omega\right)=\frac{\omega_{p}^{2}}{2 \beta^{2} \Lambda_{q_{\|}}\left(\Lambda_{q_{\|}}+q_{\|}\right)-\omega_{p}^{2}},
$$

where

$$
\Lambda_{q_{\|}}=\frac{1}{\beta} \sqrt{\omega_{p}^{2}+\beta^{2} q_{\|}^{2}-\omega(\omega+i \eta)}
$$

and $\beta$ represents the speed of propagation of hydrodynamic disturbances in the electron system.47

Within a quantized hydrodynamic model, one first linearizes the Hamiltonian of the hydrodynamic system with respect to the induced electron density, and then quantizes this Hamiltonian on the basis of the normal modes of oscillation, which are referred after quantization as bulk and surface plasmons. Hence, within this approach one can distinguish the separate contributions to the energy-loss function $\operatorname{Im} g\left(q_{\|}, \omega\right)$ coming from the excitation of either bulk or surface plasmons: 48

$$
\begin{aligned}
\operatorname{Im} g^{B}\left(q_{\|}, \omega\right)= & \frac{1}{2} q_{\|} \int_{0}^{\infty} d q_{z} \delta\left(\omega-\omega_{q}^{B}\right) \\
& \times \frac{\left(\omega_{p}^{2} / \omega_{q}^{B}\right) q_{z}^{2}}{q_{z}^{4}+q_{z}^{2}\left(q_{\|}^{2}+\omega_{p}^{2} / \beta^{2}\right)+\omega_{p}^{4} /\left(4 \beta^{4}\right)}
\end{aligned}
$$

and

$$
\operatorname{Im} g^{S}\left(q_{\|}, \omega\right)=\frac{\pi}{2} \frac{\gamma_{q_{\|}}}{q_{\|}+2 \gamma_{q_{\|}}} \frac{\omega_{p}^{2}}{\omega_{q_{\|}}^{S}} \delta\left(\omega-\omega_{q_{\|}}^{S}\right),
$$

respectively. Here, $\omega_{q}^{B}$ and $\omega_{q_{\|}}^{S}$ represent the dispersion of bulk and surface plasmons,

$$
\left(\omega_{q}^{B}\right)^{2}=\omega_{p}^{2}+\beta^{2} q^{2}
$$

and

$$
\left(\omega_{q_{\|}}^{S}\right)^{2}=\frac{1}{2}\left[\omega_{p}^{2}+\beta^{2} q_{\|}^{2}+\beta q_{\|} \sqrt{2 \omega_{p}^{2}+\beta^{2} q_{\|}^{2}}\right] .
$$

As in Eq. (19) $q=\sqrt{q_{\|}^{2}+q_{z}^{2}}$, and

$$
\gamma_{q_{\|}}=\frac{1}{2 \beta}\left(-\beta q_{\|}+\sqrt{2 \omega_{p}^{2}+\beta^{2} q_{\|}^{2}}\right) .
$$

For the separate contributions to the sum-rule of Eq. (23) coming from bulk and surface plasmons, integration of Eqs. (41) and (42) yields

$$
\int_{0}^{\infty} d \omega \omega \operatorname{Im} g^{B}\left(q_{\|}, \omega\right)=\frac{\pi}{4} \frac{q_{\|}}{q_{\|}+2 \gamma_{q_{\|}}} \omega_{p}^{2}
$$

and

$$
\int_{0}^{\infty} d \omega \omega \operatorname{Im} g^{S}\left(q_{\|}, \omega\right)=\frac{\pi}{4} \frac{2 \gamma_{q_{\|}}}{q_{\|}+2 \gamma_{q_{\|}}} \omega_{p}^{2},
$$

respectively. It is then straightforward to show that for a semi-infinite system with a uniform electron density $\bar{n}$ that drops abruptly to zero at the surface the sum of bulk and surface contributions to the energy-loss function $\operatorname{Im} g\left(q_{\|}, \omega\right)$ satisfies Eq. (23).

In the limit as $q_{\|} \rightarrow 0$ the bulk contribution to the energy-loss function vanishes, and both the imaginary part of Eq. (39), on the one hand, and Eq. (42), on the other hand, yield the long-wavelength limit of Eq. (24).

\section{Specular reflexion model}

Either by neglecting, within the IBM, the interference between incident and scattered electrons (CIBM), 15 or by simply assuming that electrons are specularly reflected at the surface (SRM) 46 , one finds 


$$
g\left(q_{\|}, \omega\right)=\frac{1-\epsilon_{s}\left(q_{\|}, \omega\right)}{1+\epsilon_{s}\left(q_{\|}, \omega\right)}
$$

where

$$
\epsilon_{s}\left(q_{\|}, \omega\right)=\frac{q_{\|}}{\pi} \int_{-\infty}^{\infty} \frac{d q_{z}}{q^{2}} \epsilon^{-1}(q, \omega),
$$

with $q=\sqrt{q_{\|}^{2}+q_{z}^{2}}$ and $\epsilon^{-1}(q, \omega)$ being the inverse bulk dielectric function of Eq. (20).

If dispersion effects are neglected altogether, thereby replacing the momentum-dependent dielectric function $\epsilon(q, \omega)$ entering Eq. (49) by a local dielectric function $\epsilon(\omega)$, Eq. (48) yields the classical prediction [Eq. (35)]. Alternatively, if dispersion effects are incorporated in an approximated manner through the hydrodynamic dielectric function of a uniform electron gas,

$$
\epsilon(q, \omega)=1+\frac{\omega_{p}^{2}}{\beta^{2} q^{2}-\omega(\omega+i \eta)},
$$

Eq. (48) is easily found to yield the hydrodynamic surface-response function [Eq. (39)].

\section{RESULTS AND DISCUSSION}

We choose the bulk charge density $\bar{n}$ to be equal to the average electron density of valence electrons in aluminum metal $\left(r_{s}=2.07\right)$, for which the Fermi momentum $\left[q_{F}=\left(3 \pi^{2} \bar{n}\right)^{1 / 3}\right]$ and bulk plasma frequency $\left[\omega_{p}\right]$ are $q_{F}=0.927 a_{0}^{-1}\left(a_{0}\right.$ is the Bohr radius, $\left.a_{0}=0.529 \AA\right)$ and $\omega_{p}=15.8 \mathrm{eV}$, respectively. We set $Z_{1}= \pm 1$ and our results can then be used for arbitrary values of $Z_{1}$, as the energy-loss probability is, within linear-response theory, proportional to $Z_{1}^{2}$.

In this section, we first show results for the energy-loss function $\operatorname{Im} W\left(z, z ; q_{\|}, \omega\right)$ entering Eqs. (15) and (17). Fig. 1 shows $\operatorname{Im} W\left(z, z ; q_{\|}, \omega\right)$, as a function of $\omega$, with $q_{\|}=0.4 q_{F}$ (for this small value of $q_{\|}$both bulk and surface plasmons are well defined excitations) and $z$ coordinates that are either well inside the solid $\left[z \leq-\lambda_{F}\right]$ (Fig. 1a) or far from the surface into the vacuum $\left[z \geq \lambda_{F}\right]$ (Fig. 1b). We have carried out self-consistent RPA and ALDA slab calculations for this quantity, as described in Sec. II.B, and have found the expected result that for these values of $z$ they coincide with those obtained from Eqs. (19) $\left[z \leq-\lambda_{F}\right]$ and (21) $\left[z \geq \lambda_{F}\right]$. Hence, the energy-loss function $\operatorname{Im} W\left(z, z ; q_{\|}, \omega\right)$ represented in Fig. 1 either does not depend on $z$ (Fig. 1a) or depends on $z$ through an overall factor of $\mathrm{e}^{-2 q_{\|} z}$ (Fig. 1b).

In Fig. 1a, bulk-plasmon (dashed line) and electronhole-pair (dotted line) contributions to the RPA energyloss function $\operatorname{Im} W\left(z, z ; q_{\|}, \omega\right)$ are shown separately, as obtained from Eq. (19), together with the total energyloss function represented by a solid line. One sees that inside the solid the energy-loss spectrum is dominated, for small values of $q_{\|}$, by a continuum of bulk-plasmon excitations occurring at energies $\omega_{q_{\|}}^{B}<\omega<\omega_{q_{c}}^{B}$, where $q_{c}$ represents the critical momentum for which the bulkplasmon dispersion $\omega_{q}^{B}$ enters the electron-hole-pair excitation spectrum. For $r_{s}=2.07$ and $q_{\|}=0.4 q_{F}$, one finds $\omega_{q_{\|}}^{B}=17.6 \mathrm{eV}$ and $\omega_{q_{c}}^{B}=23.6 \mathrm{eV}$, and bulk plasmons can be excited by charged particles moving parallel with the surface with speed $v>1.13 v_{0}\left(v_{0}\right.$ is the Bohr velocity, $\left.v_{0}=2.19 \times 10^{6} \mathrm{~ms}^{-1}\right)$.

For $z$ coordinates that are outside the surface it had been generally believed that the energy loss originates entirely in the excitation of surface plasmons and electron-hole pairs.49 Nevertheless, the continuum of bulk-plasmon excitations dominating the energy loss of charged particles moving inside the solid (see Fig. 1a) is still present for $z$ coordinates outside the surface, as shown in Fig. 1b, although the main contribution to the energy loss now clearly comes from the excitation of surface plasmons at $\omega=\omega_{q_{\|}}^{S}$ [for $r_{s}=2.07$ and $q_{\|}=0.4 q_{F}$, one finds $\left.\omega_{q_{\|}}^{S} \sim 16.0 \mathrm{eV}\right]$. The bulk-plasmon contribution to $\operatorname{Im} W\left(z, z, q_{\|}, \omega\right)$, as obtained for $z=\lambda_{F}$ within a quantized hydrodynamic model by introducing Eq. (41) into Eq. (21) (see also Ref. 48), is represented in Fig. $1 \mathrm{~b}$ by a dotted line. The total SRM energy-loss function, as obtained with the use of Eq. (48) and the RPA dielectric function $\epsilon(q, \omega)$, is represented in Fig. 1b by a solid line. This curve shows that at low frequencies the energy-loss spectrum is dominated by the creation of electron-hole pairs, losses centered around $\omega_{q_{\|}}^{S}$ are due to the excitation of surface plasmons exhibiting a finite linewidth, and bulk-plasmon excitations yield energy losses at $\omega \geq \omega_{q_{\|}}^{B}$ that nearly coincide with the result one obtains within the quantized hydrodynamic model. We have also carried out self-consistent slab calculations of the energy-loss function $\operatorname{Im} g\left(q_{\|}, \omega\right)$, which for small values of $q_{\|}\left(q_{\|}<q_{F}\right)$ has been found to be close to that obtained within the SRM. Nevertheless, differences have been found in the surface-plasmon energy $\omega_{q_{\|}}^{S}$, which shifts to lower frequencies as demonstrated earlier 50,51 also, the thickness of the slab is required to be very large for the self-consistent calculations to properly account for the high-energy spectrum originated in the excitation of bulk plasmons and electron-hole pairs.

Fig. 2 shows self-consistent calculations of the probability $P(\omega)$ for a charged particle to exchange energy $\omega$ with the medium, as obtained from Eq. (17). The particle is assumed to move parallel to the surface with $v=2 v_{0}$ and two different $z$ coordinates for which the electronic selvage at the surface is expected to play an important role: $z=-\lambda_{F} / 2$ (Fig. 2a) and $z=\lambda_{F} / 2$ (Fig. $2 \mathrm{~b})$. Dashed, thick-solid and thin-solid lines give the result of IBM, RPA and ALDA calculations, and dasheddotted lines represent the SRM probability obtained with the RPA dielectric function $\epsilon(q, \omega)$.

In the interior of the solid (Fig. 2a), the bulk-plasmon energy-loss spectrum is known to be inhibited by the presence of surface-plasmon losses through the so-called 
bregenzung or boundary effect predicted by Ritchie, whose existence is due to the orthogonality of the surfaceplasmon and bulk-plasmon modes. Nevertheless, as the electronic selvage is changed from zero (SRM) to its actual structure (RPA and ALDA) 52 the creation of electron-hole pairs increases, the surface-plasmon peak diminishes, and a broad bulk-plasmon peak dominates the spectrum, showing that a proper treatment of the surface density-profile is crucial for the energy dependence of the energy-loss probability. These results are in qualitative agreement with the calculations reported in Ref. 21, where the selvage structure is introduced in an approximate manner within the HD and the SR models.

Bulk-plasmon losses occurring in the vacuum side of the surface (see Fig. 1b) are not visible in the total energy-loss probability $P(\omega)$ which is dominated by the excitation of surface plasmons and electron-hole pairs, as shown in Fig. 2b. Also, this figure shows substantial changes in the energy-loss probability as a realistic description of the surface response is considered, with an important shift of the surface-plasmon peak towards smaller energies, in agreement with the experimental y determined surface-plasmon energies of simple metals.50.51

Figure 3 shows a surface plot of our full IBM (Fig. 3a), RPA (Fig. 3b) and ALDA (Fig. 3c) calculations of the probability $P(\omega)$ for a charged particle moving with speed $v=2 v_{0}$ parallel to the surface. The plot is shown as a function of the energy loss $\omega$ and the distance $z$ from the particle trajectory to the surface. Although the energy-loss probability is found to be divided into losses centered around the bulk-plasmon energy (in the interior of the solid) and the surface-plasmon energy (outside the solid), this separation is not as clear as predicted with the use of simplified models for the surface response (see, e.g., Ref. 21). As $z \rightarrow-\infty$ the energy-loss probability $P(\omega)$ reaches a constant shape centered around the bulk-plasmon energy, which does not depend on the details of the electronic selvage at the surface and only depends on whether the xc kernel $f_{x c}\left(z, z^{\prime} ; q_{\|}, \omega\right)$ is set equal to zero (IBM and RPA) or not (ALDA). Outside the solid, the energy-loss probability, which is centered around the surface-plasmon energy, decreases with the distance $z$ from the surface to the particle trajectory.

Fig. 4a depicts our full IBM (dashed line), RPA (thicksolid line) and ALDA (thin-solid line) calculations of the stopping power, as obtained from either Eq. (14) or (16) as a function of $z$ and with $v=2 v_{0}$. In the interior of the solid, where the electron density is taken to be constant, both IBM and RPA stopping powers coincide with the well-known RPA stopping power of a uniform electron gas. Short-range xc effects, included in the ALDA, provoke a reduction in the screening of electron-electron interactions, thereby increasing the energy loss. Outside the solid the electronic selvage at the surface plays a crucial role in the actual behaviour of the stopping power: a slow decrease of the electron density at the metal surface leads to a larger energy-loss probability [see also Fig. 2 and Eq. (23)], and the IBM stopping power is, therefore, found to be too small. In the SRM the electron density is assumed to drop abruptly to zero at the surface, which provokes a reduction in the electron-hole excitation probability, and the stopping power outside the solid (see Fig. 4b) is found to be even smaller than in the IBM. Low-energy excitations involve transitions from occupied electronic states near the Fermi level, which are sensitive to the actual density profile at the surface, and are found to play an important role in the energy-loss mechanism of charged particles moving with $v \leq 2 v_{0}$. Also plotted in Fig. $4 \mathrm{~b}$ is the result of assuming that the stopping power for a charged particle that moves at a distance $z$ from the surface can be approximated by that of a uniform electron gas with the local density $n(z) .53$ This often-used local-density approximation also yields an inaccurate description of the position-dependent stopping power, due to the intrinsic nature of surface-induced excitations not present within this approach, and the results presented in Fig. 4 show the need for a self-consistent description of the surface response if one is to look at the energy loss of charged particles moving outside a solid surface.

As the velocity increases the energy-loss spectrum of charged particles moving far from the surface into the vacuum is dominated by long-wavelength excitations and the stopping power is dictated by the integration of $\omega \operatorname{Im} g\left(q_{\|}, \omega\right)$, which as a result of particle conservation [see Eq. 23)] does not depend on the details of the actual response of the solid. In this limit and with the aid of Eq. (23) one easily finds the classical stopping power of Eq. 37 .

The velocity dependence of the stopping power is shown in Fig. 5a, for a particle moving outside the surface at $z=\lambda_{F}$. Our full IBM, RPA and ALDA calculations are represented, as in Fig. 4a, by dashed, thick-solid and thin-solid lines, respectively, and the SRM stopping power is represented by a dashed-dotted line, as obtained with the RPA dielectric function $\epsilon(q, \omega)$. At low velocities the energy-loss spectrum is dominated by intermediate and short-wavelength excitations, even far from the surface into the vacuum, and a combination of the actual electronic selvage at the surface with the intrinsic nature of surface-induced excitations play an important role in increasing the energy-loss. At high velocities the energyloss spectrum is dominated by the surface-plasmon excitation [see Eq. (24)] and all calculations converge with the classical limit of Eq. (37), as shown in Fig. 5b. As in Fig. 4b, the local-density approximation is also represented in this figure, showing that this often-used approximation cannot account for the energy loss originated in surface-induced excitations, not even at low velocities where the energy loss is entirely due to the excitation of electron-hole pairs. 


\section{SUMMARY}

We have reported self-consistent calculations of the energy loss spectra of charged particles moving parallel to a plane-bounded free-electron gas, in the framework of linear-response theory.

We have found that the continuum of bulk-plasmon excitations dominating the energy loss of charged particles moving inside the solid is still present for particle trajectories outside the surface. Nevertheless, these bulkplasmon excitations are found not to be visible in the total energy-loss probability $P(\omega)$ which outside the solid is clearly dominated by the excitation of surface plasmons and electron-hole pairs.

As for the effect of the electronic selvage at the surface, we have found that the so-called bregenzung or boundary effect inside the solid is diminished, plasmon peaks are broadened, and the surface-plasmon peak is considerably shifted towards smaller energies. The electronic selvage at the surface has also been found to increase both the energy-loss probability and the stopping power for charged particles moving in the vacuum side of the surface.

In the high-velocity limit and for charged particles moving far from the surface into the vacuum the actual stopping power is found to converge with the classical limit dictated by Eq. (37). However, at low and intermediate velocities substantial changes in the stopping power have been observed as a realistic description of the surface response is considered, and we have concluded that a self-consistent description of the surface response is necessary if one is to look at the energy loss of charged particles moving outside a solid surface. Accurate measurements of the energy loss of protons being reflected from a variety of sglid surfaces at grazing incidence have been reported. 46 A theoretical description of these experiments requires that the ion trajectory $z(t)$ be calculated and energy losses from the excitation of innershells be taken into account. Also, in real experiments band-structure effects might be important and the surface roughness might lead to additional energy loss due to the so-called Smith-Purcell effect.57 Work in this direction is now in progress.

\section{ACKNOWLEDGMENTS}

The authors would like to thank A. G. Eguiluz and R. H. Ritchie for useful discussions in connection with this research. Partial support by the University of the Basque Country, the Basque Unibertsitate eta Ikerketa Saila, and the Spanish Ministerio de Educación y Cultura is also acknowledged.

\section{APPENDIX A:}

Here we give explicit expressions for the screened interaction and the surface-response function in terms of the Fourier coefficients of the density-response function.

We first introduce the following double-cosine Fourier representation for the density-response function:

$$
\chi\left(z, z^{\prime} ; q_{\|}, \omega\right)=\sum_{m=0}^{\infty} \sum_{n=0}^{\infty} \chi_{m n}\left(q_{\|}, \omega\right) \cos (m \pi \tilde{z}) \cos \left(n \pi \tilde{z}^{\prime}\right),
$$

where $d=a+2 z_{0}$ and $\tilde{z}=\left(z+z_{0}+a\right) / d$.

Introducing Eq. (A1) into Eq. (18), we obtain the following expression for the screened interaction:

$$
W\left(z, z^{\prime} ; q_{\|}, \omega\right)=\sum_{m=0}^{\infty} \sum_{n=0}^{\infty} W_{m n}\left(q_{\|}, \omega\right) \cos (m \pi \tilde{z}) \cos \left(n \pi \tilde{z}^{\prime}\right)
$$

where

$$
\begin{aligned}
W_{m n}\left(q_{\|}, \omega\right) & =v_{m n}\left(q_{\|}\right)+\frac{\mu_{m} \mu_{n}}{d^{2}} \\
\times & \sum_{m^{\prime}=0}^{\infty} \sum_{n^{\prime}=0}^{\infty} v_{m m^{\prime}}\left(q_{\|}\right) \chi_{m^{\prime} n^{\prime}}\left(q_{\|}, \omega\right) v_{n^{\prime} n}\left(q_{\|}\right), \\
v_{m n}\left(q_{\|}\right)= & \frac{2 \pi e^{2}}{q_{\|}^{2}+(m \pi / d)^{2}}\left[\frac{2 d}{\sqrt{\mu_{m} \mu_{n}}} \delta_{m n}\right. \\
& \left.-\left[1+(-1)^{m+n}\right] \frac{q_{\|}\left[1-(-1)^{m} \mathrm{e}^{-q_{\|}} d\right]}{\left(q_{\|}^{2}+(n \pi / d)^{2}\right.}\right],
\end{aligned}
$$

and

$$
\mu_{m}= \begin{cases}1, & \text { for } m=0 \\ 2, & \text { for } m \geq 1\end{cases}
$$

Similarly, introduction of Eq. (A1) into Eq. (22) yields:

$g\left(q_{\|}, \omega\right)=-\frac{2 \pi d^{2}}{q_{\|}} \sum_{m=0}^{\infty} \sum_{n=0}^{\infty} \frac{1}{\mu_{m} \mu_{n}} \alpha_{m} \alpha_{n} \chi_{m n}\left(q_{\|}, \omega\right)$,

where

$$
\alpha_{m}=-\frac{\mu_{m} q_{\|}}{d} \frac{1-e^{q_{\|} d} \cos (m \pi)}{q_{\|}^{2}+(m \pi / d)^{2}} .
$$

${ }^{1}$ D. Pines, Solid State Phys. 1, 367 (1955). 
${ }^{2}$ R. H. Ritchie, Phys. Rev. 106, 874 (1957).

${ }^{3}$ H. Raether, in Excitations of Plasmons and Interband Transitions by Electrons, edited by G. Höhler, Springer Tracks in Modern Physics, Vol. 88 (Springer, Berlin, 1980).

${ }^{4}$ P. E. Batson, Phys. Rev. Lett. 49, 936 (1982).

${ }^{5}$ H. Kohl, Ultramicroscopy 11, 53 (1983).

${ }^{6}$ R. H. Ritchie and A. Howie, Philos. Mag. A 58, 753 (1988).

${ }^{7}$ D. W. McComb and A. Howie, Nucl. Instrum. Methods B 96, 569 (1995).

${ }^{8}$ D. H. Schneider and M. A. Briere, Phys. Scr. 53, 228 (1996).

${ }^{9}$ A. Arnau et al, Surf. Sci. Rep. bf 27, 113 (1997).

${ }^{10} \mathrm{H}$. Winter, M. Wilke, and M. Bergomaz, Nucl. Instrum. Methods B 125, 124 (1997).

${ }^{11}$ D. Niemman, M. Grether, A. Spieler, N. Stolterfoht, C. Lemell, F. Aumayr, and H. P. Winter, Phys. Rev. A 56, 4774 (1997).

12 D. Niemman, M. Grether, M. Rösler, and N. Stolterfoht, Phys. Rev. Lett. 80, 3328 (1998).

${ }^{13}$ M. Hattas, T. Schenkel, A. V. Hamza, A. V. Barnes, M. W. Newman, J. W. McDonald, T. R. Niederwayr, G. A. Machicoane, and D. H. Schneider, Phys. Rev. Lett. 82, 4795 (1999).

${ }^{14}$ P. M. Echenique and J. B. Pendry, J. Phys. C 8, 2936 (1975).

${ }^{15}$ R. Nuñez, P. M. Echenique, and R. H. Ritchie, J. Phys. C 13, 4229 (1980).

${ }^{16}$ N. Zabala and P. M. Echenique, Ultramicroscopy 32, 327 (1990).

${ }^{17}$ F. Yubero and S. Tougaard, Phys. Rev. B 46, 2486 (1992).

${ }^{18}$ Y. F. Chen and Y. T. Chen, Phys. Rev. B 53, 4980 (1996).

19 J. I. Juaristi, F. J. García de Abajo, and P. M. Echenique, Phys. Rev. B 53, 13839 (1996).

${ }^{20}$ Z. J. Ding, Phys. Rev. B 55, 9999 (1997); J. Phys.:Condens. Matter 10, 1733 (1998); J. Phys.:Condens. Matter 10, 1753 (1998).

21 T. Nagatomi, R. Shimizu, and R. H. Ritchie, Surf. Sci. 419, 158 (1999). Methods B 125, 106 (1997).

${ }^{22}$ M. A. Cazalilla and F. J. Garcia de Abajo, Nucl. Instrum.

${ }^{23}$ J. M. Pitarke, R. H. Ritchie, and P. M. Echenique, Nucl. Instrum. Methods B 79, 209 (1993).

${ }^{24}$ J. M. Pitarke, R. H. Ritchie, P. M. Echenique, and E. Zaremba, Europhys. Lett. 24, 613 (1993); J. M. Pitarke, R. H. Ritchie, and P. M. Echenique, Phys. Rev. B52, 13883 (1995).

25 A. Bergara, J. M. Pitarke, and R. H. Ritchie, Phys. Rev. B 60, 16176 (1999).

26 This approximation is valid for heavy charged particles, e.g., ions, and also for swift electrons moving with velocities that are large compared to those of the electrons in the medium. In the case of electrons, $Z_{1}=-1$.

27 J. M. Pitarke and I. Campillo, Nucl. Instrum. Methods B 64, 141 (2000).

${ }^{28} \mathrm{P}$. Nozieres and D. Pines, The theory of quantum liquids (Benjamin, New York, 1966).

${ }^{29}$ P. M. Echenique, J. M. Pitarke, E. V. Chulkov, and A. Rubio, Chem. Phys. 251, 1 (2000).

${ }^{30} \mathrm{~F}$. Flores and F. García Moliner, Introduction to the Theory of Solid Surfaces (Cambridge University Press, Cambridge, 1979).
${ }^{31}$ D. S. Gemmell, Rev. Mod. Phys. 46, 129 (1974).

${ }^{32}$ A. Liebsch, Electronic excitations at metal surfaces (Plenum Press, New York, 1997).

${ }^{33}$ E. K. U. Gross, J. F. Dobson, and M. Petersilka, in Density Functional Theory II, Vol. 181 of Topics in Current Chemistry, edited by R. F. Nalewajski (Springer, Berlin, 1996).

${ }^{34}$ P. Hohenberg and W. Kohn, Phys. Rev. 136 B864 (1964); W. Kohn and L. J. Sham, Phys. Rev. 140, A11333 (1965).

35 J. Bardeen, Phys. Rev. 49, 653(1936); D. M. Newns, Phys. Rev. B 1, 3304 (1970).

36 This DFT-based RPA differs from the less realistic actual RPA or Hartree approximation described above, which is determined by setting both $v_{x c}(z)$ and $f_{x c}\left(z, z^{\prime} ; q_{\|}, \omega\right)$ equal to zero.

37 A. Zangwill and P. Soven, Phys. Rev. A 21, 1561 (1980).

38 A. G. Eguiluz, Phys. Rev. Lett. 51, 1907 (1983); Phys. Rev. B 31, 3303 (1985).

${ }^{39}$ In the non-self-consistent IBM, $z_{0}$ is chosen so as to ensure charge neutrality. For a self-consistent description of the density-response function, $z_{0}$ is chosen sufficiently large for the physical results to be insensitive to the precise value employed.

${ }^{40}$ F. K. Schulte, Surf. Sci. 55, 427 (1976); P. J. Feibelman, Phys. Rev. B 27, 1991 (1983).

${ }^{41}$ J. M. Pitarke and A. G. Eguiluz, Phys. Rev. B 57, 6329 (1998).

42 J. M. Pitarke and A. G. Eguiluz, Phys. Rev. B 63, 45116 (2001).

${ }^{43}$ R. H. Ritchie and R. E. Wilems, Phys. Rev. 178, 372 (1969).

${ }^{44}$ G. Barton, Rep. Prog. Phys. 42, 963 (1979).

${ }^{45}$ G. E. H. Reuter and E. H. Sondheimer, Proc. R. Soc. A 195, 336 (1948); A. Griffin and J. Harris, Can. J. Phys. 54, 1396 (1976).

${ }^{46}$ R. H. Ritchie and A. L. Marusak, Surf. Sci. 4, 234 (1966).

47 The value $\beta=\sqrt{3 / 5} q_{F}$ is usually chosen to describe processes in which high frequencies of the order of the plasma frequency are involved. See, e.g., D. Pines, Elementary Excitations in Solids (Benjamin, New York, 1963).

${ }^{48}$ A.Bergara, J.M. Pitarke and R.H. Ritchie, Phys. Lett. A 256, 405 (1999).

${ }^{49}$ See, e.g., P.M. Echenique, F. J. García de Abajo, V. H. Ponce, and M. E. Uranga, Nucl. Instrum. Meth. B 96, 583 (1995).

${ }^{50}$ K-D. Tsuei, E. W. Plummer, and P. J. Feibelman, Phys. Rev. Lett. 63, 2256 (1989); S.Suto, K-D. Tsuei, E.W. Plummer eta E.Burstein, Phys. Rev. Lett. 63, 2590 (1989).

${ }^{51}$ K-D. Tsuei, E. W. Plummer, A. Liebsch, K. Kempa, and P.Bakshi, Phys. Rev. Lett. 64, 44 (1990).

52 Self-consistent RPA and ALDA electron-densities coincide and both vary very slowly as compared to the IBM. While the IBM electron density vanishes for $z \geq(3 / 16) \lambda_{F}$, selfconsistent electron densities can only be neglected for $z>$ $\lambda_{F}$ (see, e.g., Fig. 8 of Ref. 42).

53 The use of this local-density approximation, in which one replaces $-(d E / d x)(z)$ in either either Eq. (14) or (16) by the stopping power of a uniform electron gas of density $n(z)$, should not be confused with the use of the LDA xc potential in Eqs. (30) and (33). 
${ }^{54}$ K. Kimura, M. Hasegawa, and M. Mannami, Phys. Rev. B 36, 7 (1987).

${ }^{55}$ Y. Fuji, S. Fujiwara, K. Narumi, K. Kimura, and M. Mannami, Surf. Sci. 277, 164 (1992); K. Kimura, H. Kuroda, M. Fritz, and M. Mannami, Nucl. Instrum. Methods B 100, 356 (1995).

${ }^{56}$ H. Winter, M. Wilke, and M. Bergomaz, Nucl. Instrum. Methods B 125, 124 (1997).

${ }^{57}$ S. J. Smith and E. M. Purcell, Phys. Rev. 92, 1069 (1953).

FIG. 1. The energy-loss function, $\operatorname{Im} W\left(z, z ; q_{\|}, \omega\right)$, as a function of $\omega$ with $q_{\|}=0.4 q_{F}$ and $z$ coordinates that are either well inside the solid $\left[z \leq-\lambda_{F}\right]$ or far from the surface into the vacuum $\left[z \geq \lambda_{F}\right]$. (a) The solid line represents the total RPA energy-loss function, as obtained from Eq. (19) with $z \leq-\lambda_{F}$; dashed and dotted lines represent the corresponding bulk-plasmon and electron-hole-pair contributions, respectively. (b) The solid line represents the SRM energy-loss function, as obtained for $z=\lambda_{F}$ with the use of Eq. (48) and the RPA dielectric function $\epsilon(q, \omega)$; the dotted line represents the bulk-plasmon contribution, as obtained by introducing Eq. (41) into Eq. 21.

FIG. 2. The energy-loss probability $P(\omega)$, as obtained from Eq. (17) with $v=2 v_{0}$ and two different $z$ coordinates: (a) $z=-\lambda_{F} / 2$ and (b) $z=\lambda_{F} / 2$. Dashed-dotted, dashed, thick-solid and thin-solid lines represent SRM, IBM, RPA and ALDA calculations. The SRM probabilities have been obtained with the use of the RPA dielectric function $\epsilon(q, \omega)$. The damping parameter is taken to be $\gamma=\omega_{p} / 10$.

FIG. 3. The energy-loss probability $P(\omega)$, versus the energy loss $\omega$ and the $z$ coordinate, as obtained for $v=2 v_{0}$ within the (a) IBM, (b) RPA and (c) ALDA. The solid is in the region $z<0 . \gamma=\omega_{p} / 10$.

FIG. 4. Stopping power, as obtained from either Eq. (14) or (16) as a function of $z$ and with $v=2 v_{0}$. a) Dashed, thick-solid and thin-solid lines represent IBM, RPA and ALDA calculations. b) RPA (thick-solid line), SRM -as obtained with the use of the RPA dielectric function $\epsilon(q, \omega)$ (dotted line) and local-density (dashed line) calculations. The solid is in the region $z<0 . \gamma=\omega_{p} / 10$.

FIG. 5. Stopping power, as obtained from either Eq. (14) or (16) as a function of $v$ and with $z=\lambda_{F} / 2$ a) Dashed-dotted, dashed, thick-solid and thin-solid lines represent SRM -as obtained with the use of the RPA dielectric function $\epsilon(q, \omega)$-, IBM, RPA and ALDA calculations. b) IBM (solid line), SRM (dashed-dotted line) and local-density (dashed line). The classical prediction of Eq. (37) is represented by a dotted line. $\gamma=\omega_{p} / 100$. 


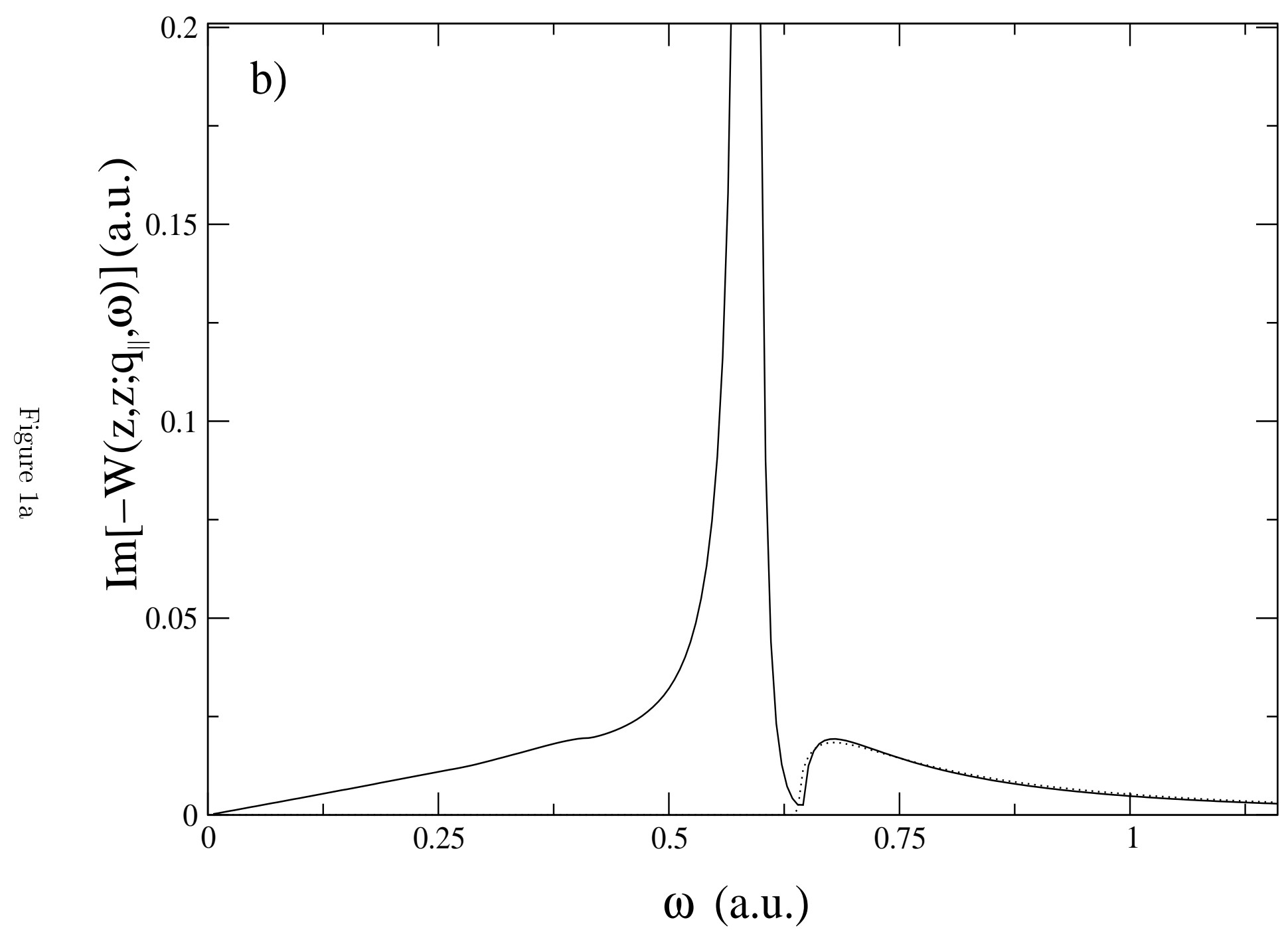




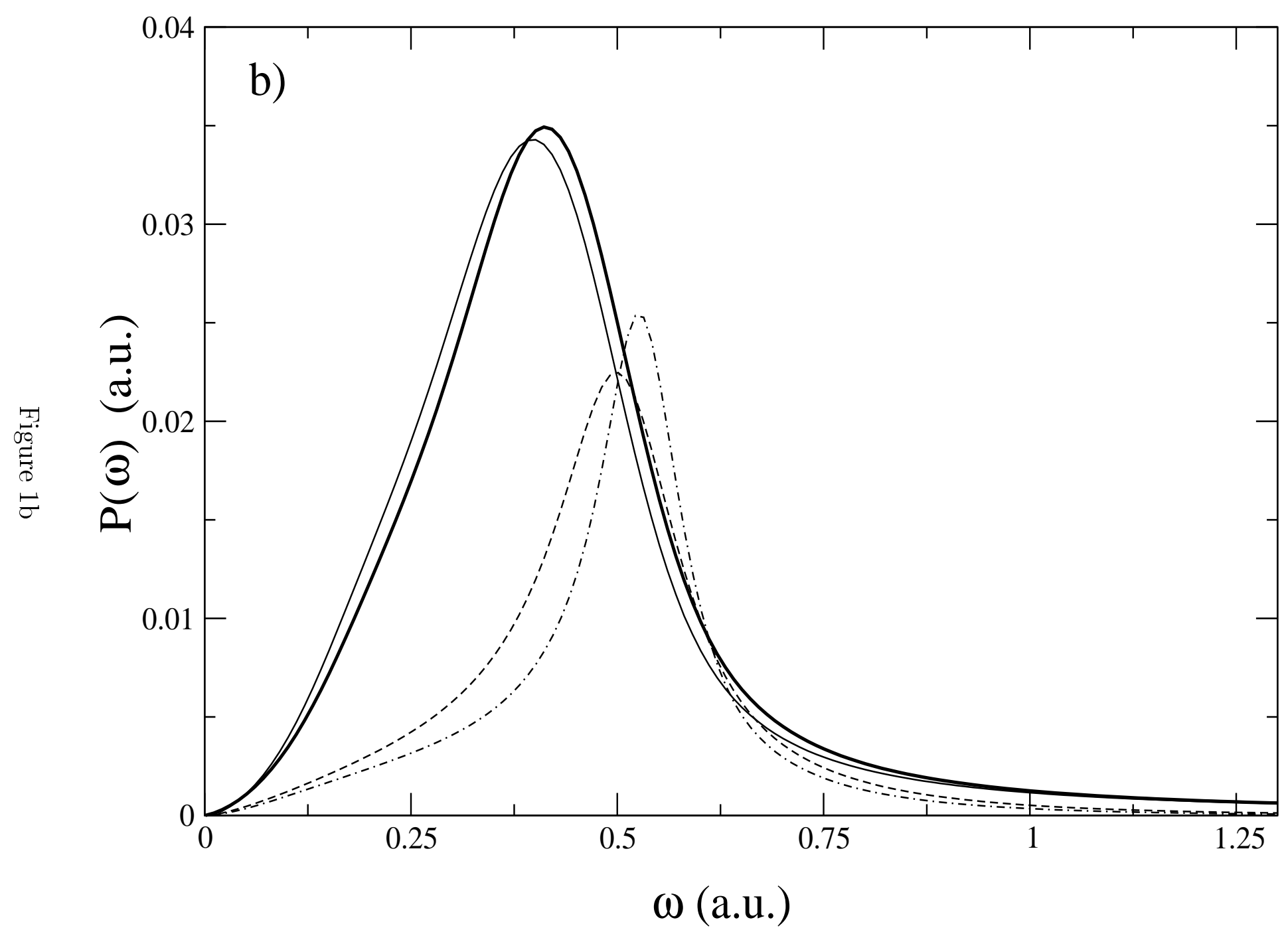




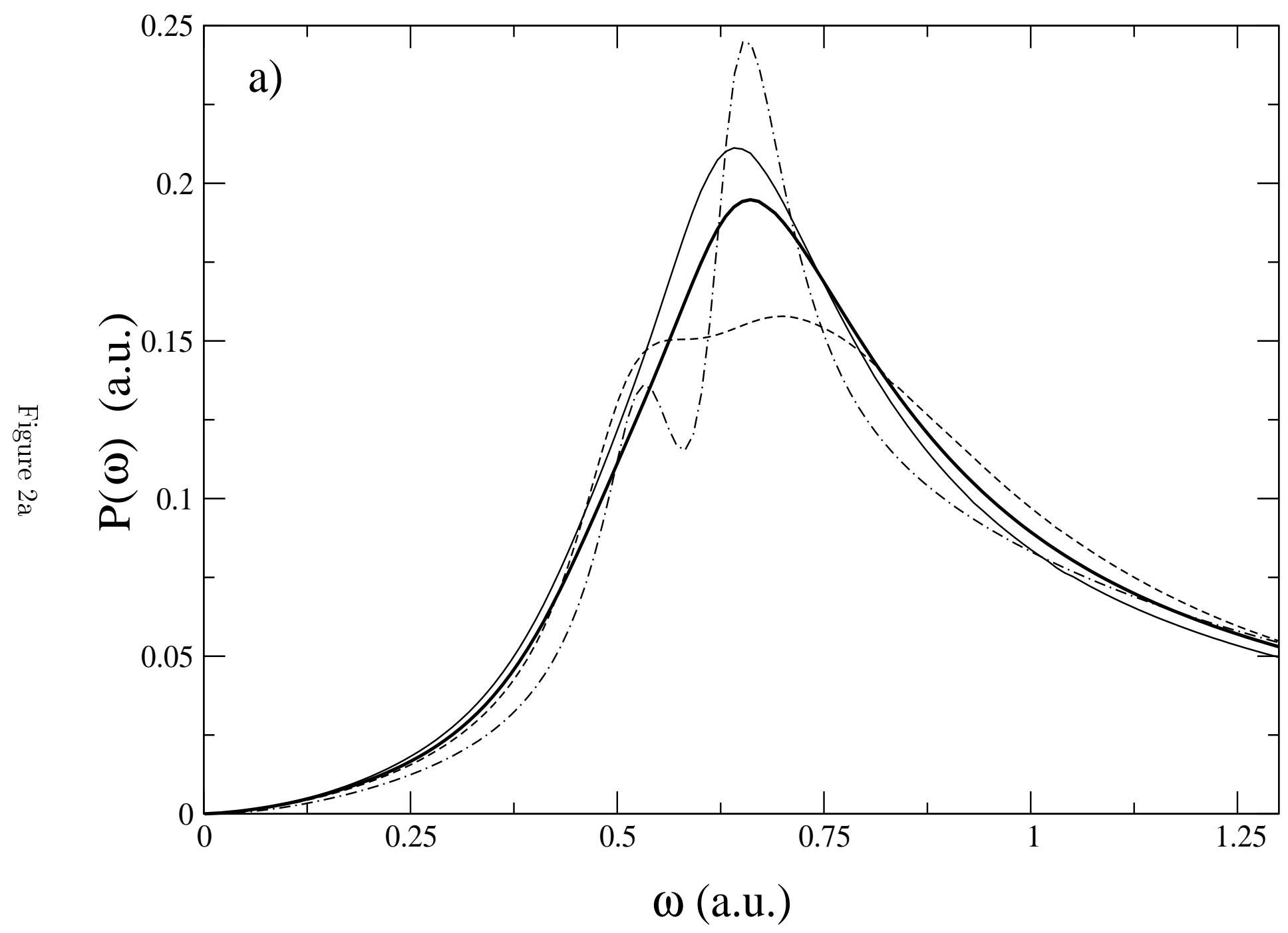




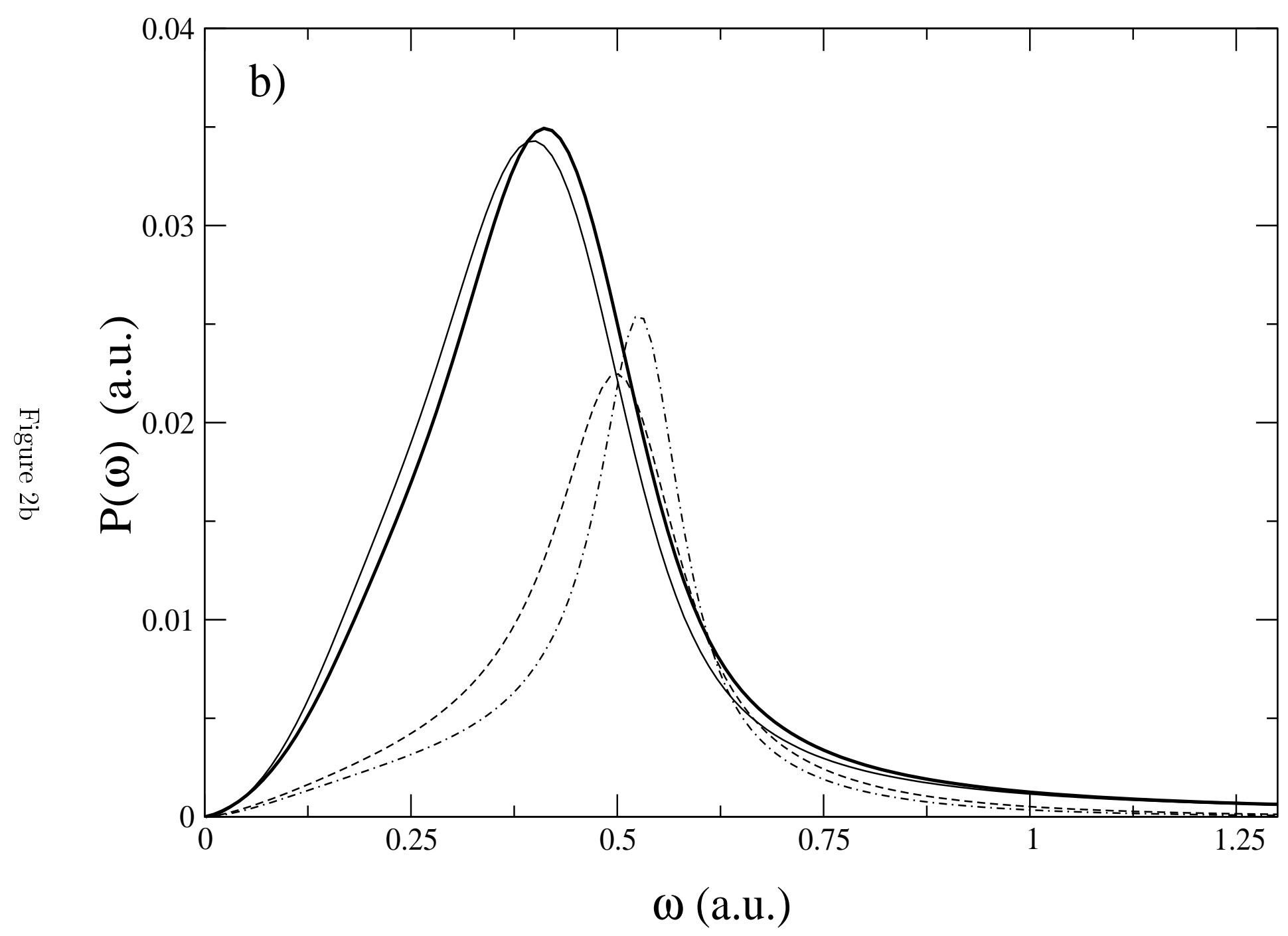


a)

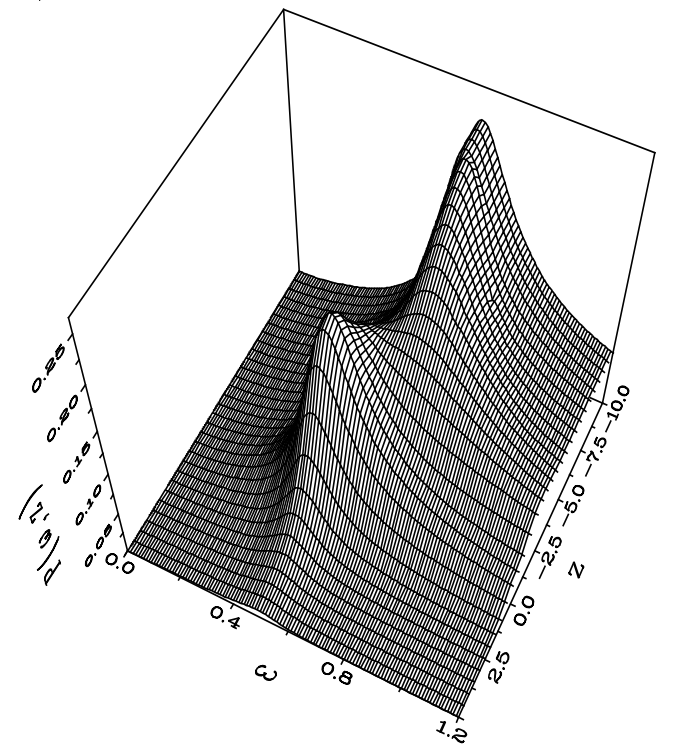

b)

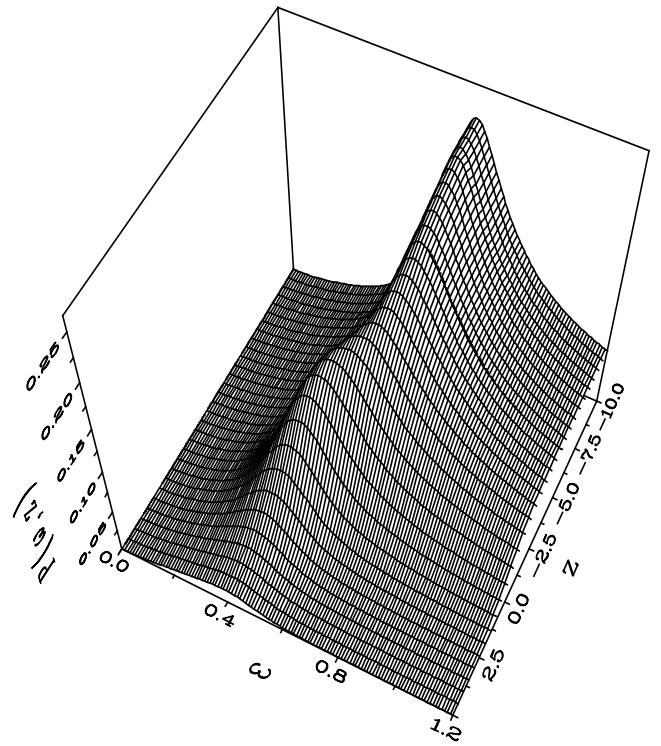

c)

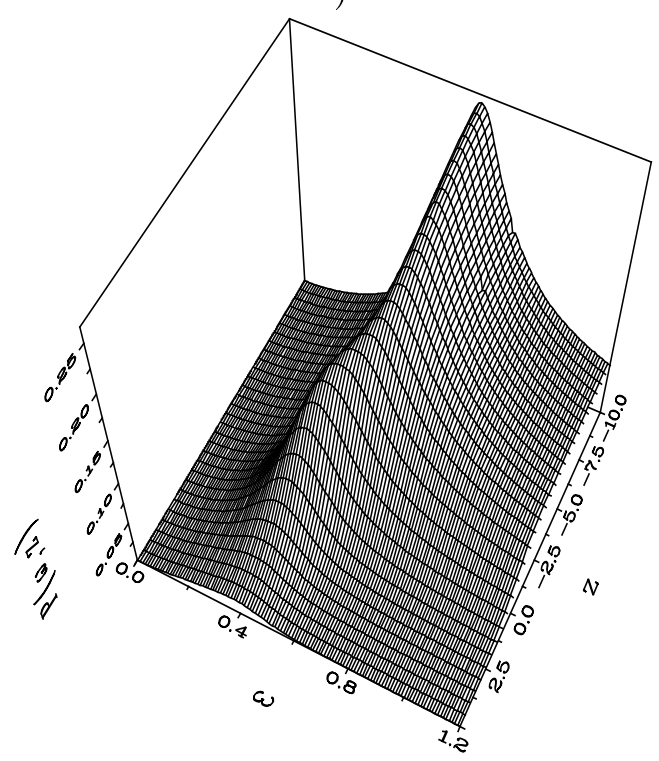

Figure 3 


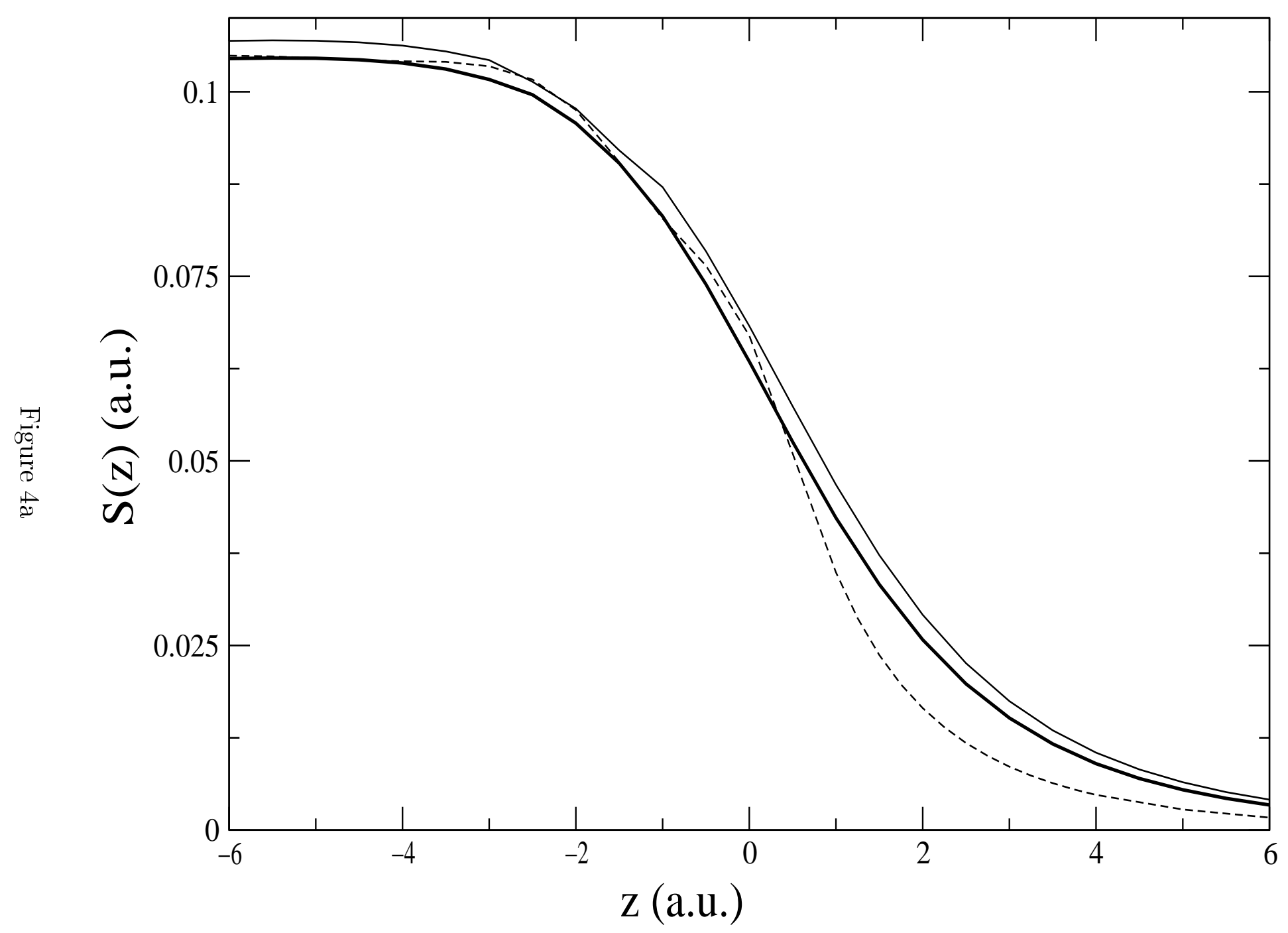




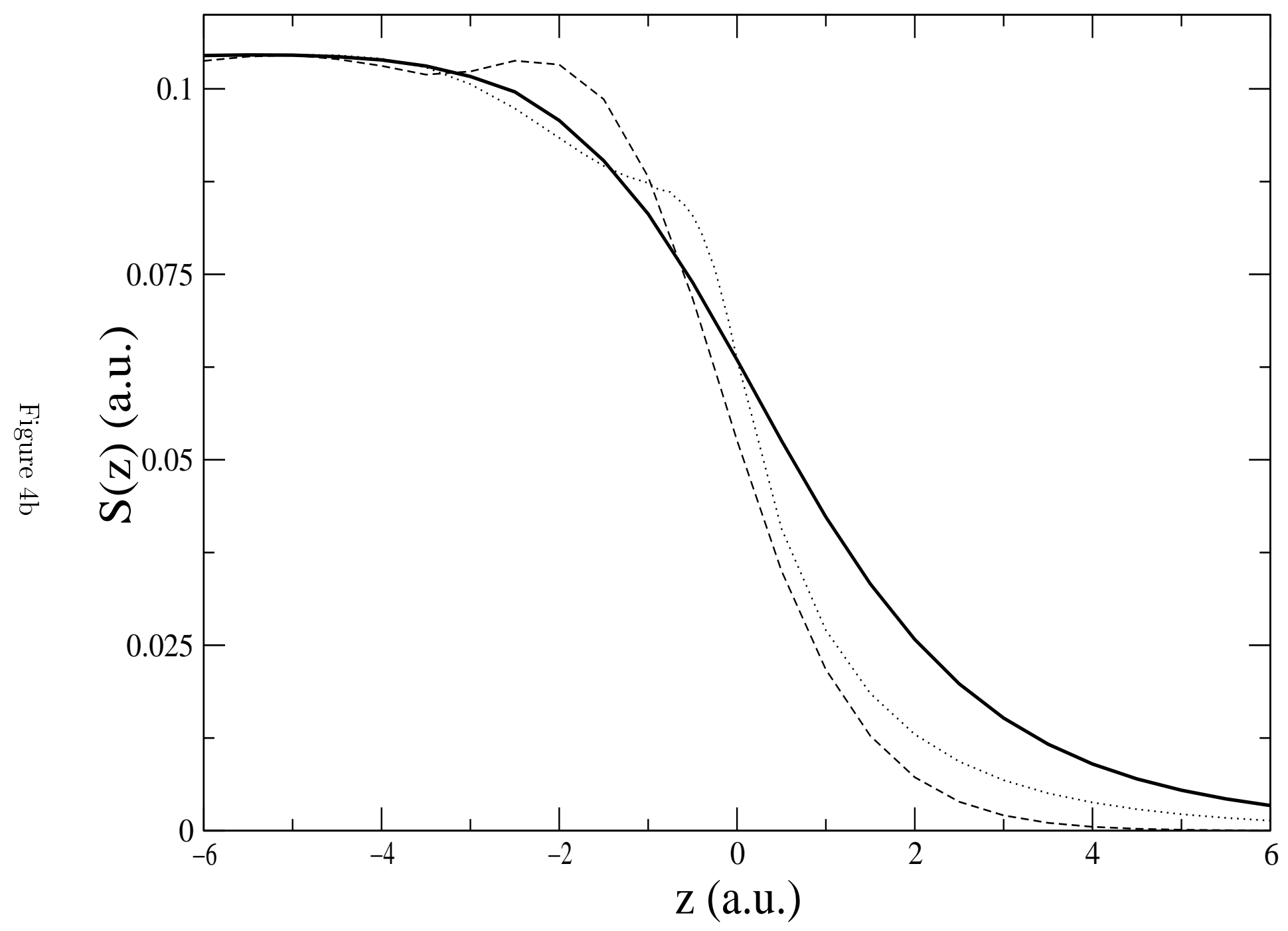




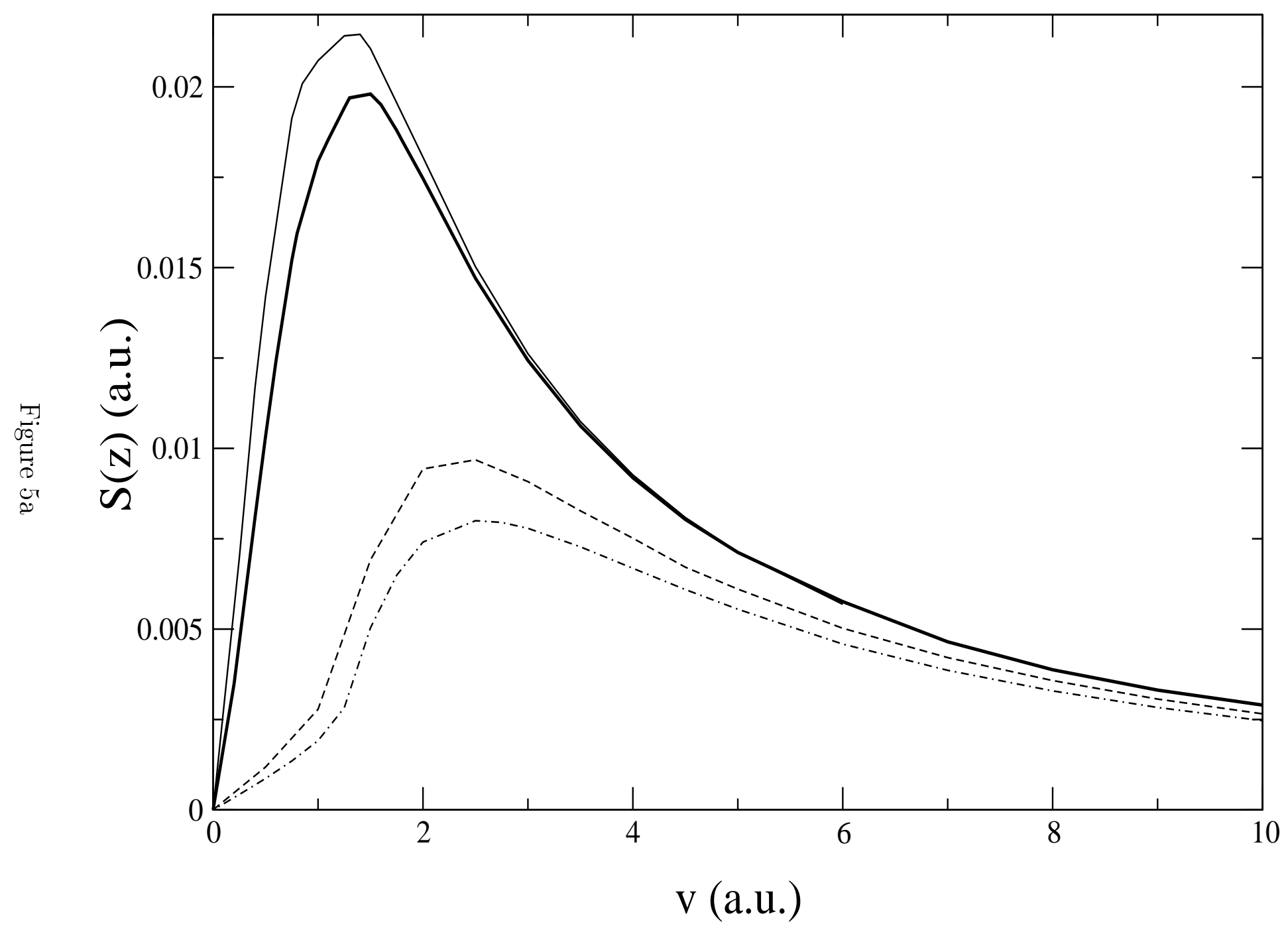




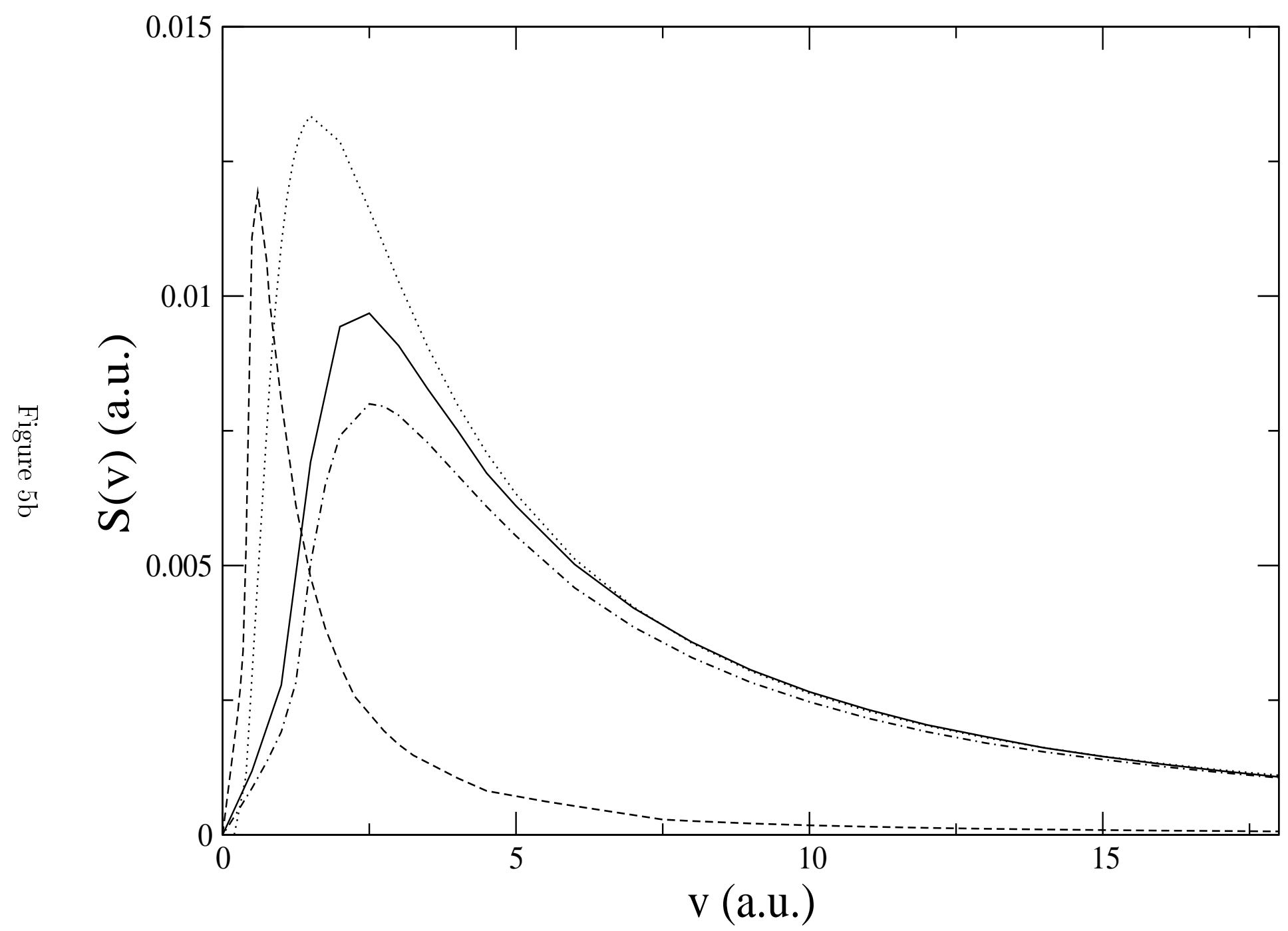

